\title{
Spontaneous emergence of rogue waves in partially coherent waves: A quantitative experimental comparison between hydrodynamics and optics
}

\author{
R. El Koussaifi, ${ }^{1,2}$ A. Tikan, ${ }^{1,2}$ A. Toffoli, ${ }^{3}$ S. Randoux,,${ }^{1,2}$ P. Suret,,${ }^{1,2}$ and M. Onorato ${ }^{4,5}$ \\ ${ }^{1}$ Laboratoire de Physique des Lasers, Atomes et Molecules, Université de Lille, UMR-CNRS 8523, 59655 Villeneuve d'Ascq, France \\ ${ }^{2}$ Centre d'Etudes et de Recherches Lasers et Applications (CERLA), 59655 Villeneuve d'Ascq, France \\ ${ }^{3}$ Department of Infrastructure Engineering, The University of Melbourne, Parkville, VIC 3010, Australia \\ ${ }^{4}$ Dipartimento di Fisica, Università degli Studi di Torino, 10125 Torino, Italy \\ ${ }^{5}$ Istituto Nazionale di Fisica Nucleare, INFN, Sezione di Torino, 10125 Torino, Italy
}

(Received 20 June 2017; published 16 January 2018)

\begin{abstract}
Rogue waves are extreme and rare fluctuations of the wave field that have been discussed in many physical systems. Their presence substantially influences the statistical properties of a partially coherent wave field, i.e., a wave field characterized by a finite band spectrum with random Fourier phases. Their understanding is fundamental for the design of ships and offshore platforms. In many meteorological conditions waves in the ocean are characterized by the so-called Joint North Sea Wave Project (JONSWAP) spectrum. Here we compare two unique experimental results: the first one has been performed in a $270 \mathrm{~m}$ wave tank and the other in optical fibers. In both cases, waves characterized by a JONSWAP spectrum and random Fourier phases have been launched at the input of the experimental device. The quantitative comparison, based on an appropriate scaling of the two experiments, shows a very good agreement between the statistics in hydrodynamics and optics. Spontaneous emergence of heavy tails in the probability density function of the wave amplitude is observed in both systems. The results demonstrate the universal features of rogue waves and provide a fundamental and explicit bridge between two important fields of research. Numerical simulations are also compared with experimental results.
\end{abstract}

DOI: 10.1103/PhysRevE.97.012208

\section{INTRODUCTION}

Ocean waves are primarily forced by the wind. After being generated, waves are subjected to nonlinear interactions, which transfer energy to different modes and thus model the ocean wave spectrum. In the late 1960s an international team of scientists carried out a field campaign with the aim of finding the shape of the wave spectrum [1]. They measured the surface elevation in 13 stations along $160 \mathrm{~km}$ in the North Sea. Frequency Fourier spectra were then computed and fitted by an empirical formula, which is known as the Joint North Sea Wave Project (JONSWAP) spectrum [2]. Except for particular meteorological or bathymetric conditions, nowadays it is well accepted by the oceanographic community that, to some extent, wind waves are described by such spectrum.

If the phases of the Fourier components of the JONSWAP spectrum are randomly distributed, the surface elevation is characterized by a Gaussian distribution, according to the central limit theorem. This implies that the envelope is Rayleigh distributed and the square of the envelope (i.e., the power) obeys the exponential distribution (see Sec. III). From a purely statistical point of view, extreme events may always take place albeit rarely. Just to give an example, according to the Rayleigh distribution, the probability of measuring a wave height that is larger that eight times the standard deviation of the surface elevation (a rogue wave) is $3.4 \times 10^{-4}$. This implies that in a storm characterized by waves that have a mean frequency of $0.1 \mathrm{~Hz}$, it would take in principle $8.3 \mathrm{~h}$ to measure such an extreme event (provided the meteo-ocean conditions remain stationary).
Due to nonlinearity, correlations of the phases can develop. Therefore, the statistical properties of the surface elevation may change and rogue waves may appear more often than predicted by the linear theory. The origin of such waves is very much debated and different explanations may be found in the literature [3-11]. In the limit of weakly nonlinear one dimensional waves, simplified forms of the primitive equation of motion may offer some insights on the problem. After the pioneering work in Refs. [12,13], it has become a common practice to investigate rogue waves using the nonlinear Schrödinger equation (NLSE) [7,8,14-17]. Inspired by the work in oceanography, the concept of rogue wave has been developed in various optical systems [18-32], starting from the work in Ref. [33]. Experiments have also been performed to generate exact solutions of the NLSE in optical fibers and water wave tanks [34-39]. These observations have been of fundamental significance for establishing the bases of a potential bridge between the fields of optics and hydrodynamics (see also Refs. [40,41] for a detailed comparison between the NLSE in optics and hydrodynamics). In most of optical fiber and water experiments, the initial conditions are deterministic and specifically designed to match peculiar exact solutions of the NLSE.

A critical challenge in the field is the development of realistic oceanic conditions in an optical fiber. The accurate measurement of the statistical properties of the wave amplitude in the fiber is also a critical issue [29-31,42,43]. Typically ocean waves are far from being either monochromatic or a supercontinuum, but they are characterized by a finite width spectrum with random phases. Such waves in statistical optics 
are known as "partially coherent waves" [44]. This is precisely the regime investigated in the present paper.

Here we report an ad hoc optical experimental set-up that has been properly designed to propagate in an optical fiber waves characterized by a JONSWAP spectrum. We quantitatively compare the evolution of the statistical properties of waves in an optical fiber against the ones recorded in the long water wave tank at Marintek, Trondheim (Norway) $[45,46]$.

By using an appropriate rescaling of the initial conditions in the optical experiments, we investigate several values of the normalized propagation length by using a given length of optical fiber. In particular this allowed us to study the statistics as a function of the propagation distance without the use of the so-called "cut-back" technique [35,47-49].

The paper is organized as follows: in Sec. II we present a unifying description of water and optical waves in the framework of NLSE; this is a very important step which allows us to design properly the experiment and scale the parameters in the optical experiment to match the hydrodynamical one. Indeed, we stress from the beginning that it is not our goal to establish quantitatively the validity of the NLSE in the respective fields or to model properly the complicated phenomena (wave breaking, friction, etc.) that may take place in a wave tank. The role played here by the NLSE is to establish the spatial and time scales over which comparison between optical and hydrodynamical experiments can be made. In Sec. III we describe some basic features of the statistical properties of linear partially coherent waves and we define the observables that will be extracted from the measurements. For the sake of completeness, in Sec. IV a quick formulation of the JONSWAP spectrum is given. In Sec. V both experimental set-ups will be described and results will be presented in Sec. VI. Conclusions will follow.

\section{A UNIFYING DESCRIPTION}

Wind waves have a typical spatial scale, $\lambda_{0}$, that ranges from a few centimeters to almost half kilometer. Those lengths corresponds approximately to frequencies of 5 to $5 \times 10^{-2}$ $\mathrm{Hz}$. Independently of the frequency of the carrier wave, $f_{0}$, the typical frequency spectral bandwidth, $\Delta f / f_{0}$, is of the order of 0.3 . Due to the relatively small wave tank $(280 \mathrm{~m})$, in our experiments we have considered waves characterized by a period of $1.5 \mathrm{~s}\left(f_{0} \sim 0.667 \mathrm{~Hz}, \lambda_{0} \sim 3.5 \mathrm{~m}\right)$. In optical fibers experiments based on telecommunications equipment, the wavelength of the carrier wave is $\lambda_{0} \sim 1.55 \mu \mathrm{m}$ that corresponds to $f_{0} \sim 2 \times 10^{14} \mathrm{~Hz}$. In optical fiber experiments involving partially coherent waves described by the NLSE, the relative spectral bandwidths are typically of the order of $0.5 \times 10^{-3}[29,30,50,51]$.

At first glance, one would be tempted to state that, due to the very different spatial and temporal scales, one would need a very short optical fiber to reproduce the wave dynamics in the water tank. As it will be clear soon, it turns out that the length of the fiber is of the same order as the length of the water wave tank.

A simple and straightforward way for appreciating this fact can be deduced by analyzing the NLSE both in optics and in water waves. While NLSE provides only an approximation to the dynamics of water waves, we have found that its use has been of practical relevance for the design of the optical experiment and for the comparison between the hydrodynamical and optical data sets. Indeed, the NLSE equation offers a common background over which nonlinear dynamics in different fields can be described $[9,40]$. For the present discussion, it is important to write the NLSE in the following form:

$$
i \frac{\partial A}{\partial z}=\frac{1}{2} \beta_{2} \frac{\partial^{2} A}{\partial t^{2}}-\chi^{(3)}|A|^{2} A,
$$

where $z$ is the propagation variable, $\beta_{2}$ and $\chi^{(3)}$ are two known constant coefficients that account for the group dispersion and the strength of nonlinearity, respectively. For water waves in infinite deep water $\chi^{(3)}=-k_{0}^{3}$ and $\beta_{2}=2 / g$, with $g$ the acceleration of gravity and $k_{0}$ the wave number of the carrier wave. In optics, in the anomalous dispersion regime (the one investigated here), $\beta_{2}$ is negative, while $\chi^{(3)}$ is always positive in fibers. It has to be mentioned that the surface elevation $\eta(x, t)$ is related at the leading order to the slowly varying complex envelope $A$ as

$$
\eta(z, t)=\frac{1}{2}\left(A(z, t) e^{i\left(k_{0} z-\omega_{0} t\right)}+\text { c.c. }\right) ;
$$

a similar relation holds for the optical (electric) field.

From Eq. (2), the following relation can be derived:

$$
\left\langle|A|^{2}\right\rangle=2\left\langle\eta^{2}\right\rangle=2 \sigma^{2},
$$

where $\langle\ldots\rangle$ implies averages over time and $\sigma^{2}$ is the variance of the rapidly oscillating wave field. While the surface elevation is directly measured in standard water wave experiments at fixed values of $z$, in optical experiments the quantity that is measured at the end of the fiber is the power that is proportional to the modulus square of the complex envelope $A$.

To design the experiment, it is useful to introduce from Eq. (1) a linear and a nonlinear propagation length in hydrodynamics and in optics as follows:

$$
z_{\text {lin }}=\frac{2}{\beta_{2} \Delta \omega^{2}} \quad \text { and } \quad z_{\text {nlin }}=\frac{1}{\chi^{(3)}\left\langle\left|A_{0}\right|^{2}\right\rangle},
$$

where $\Delta \omega=2 \pi \Delta f$ is a typical spectral bandwidth and $\left\langle\left|A_{0}\right|^{2}\right\rangle$ is the average value of the envelope square both calculated at $z=0$ that corresponds in optics to the average power $P_{0}$ injected in the fiber.

In oceanography the estimation of a characteristic amplitude is usually made by introducing the so-called significant wave height, $H_{s}$, that is defined as the average over $1 / 3$ of the highest waves in the measured time series (a wave height is the distance between a crest and the adjacent trough). Assuming Gaussian statistics for $\eta, H_{s} \simeq 4 \sigma$, with $\sigma=\sqrt{\left\langle\eta^{2}\right\rangle}$ being the standard deviation of the surface elevation [3]. Using Eq. (3), the following relation, connecting the power to the significant wave height, can be obtained straightforwardly:

$$
\left\langle\left|A_{0}\right|^{2}\right\rangle=P_{0}=H_{s}^{2} / 8 \text {. }
$$

The degree of nonlinearity of the wave propagation is given by the parameter:

$$
\epsilon=\frac{z_{\text {lin }}}{z_{\mathrm{nlin}}}=\frac{2 \chi^{(3)}\left\langle\left|A_{0}\right|^{2}\right\rangle}{\beta_{2} \Delta \omega^{2}} .
$$


In the context of ocean waves, $\sqrt{\epsilon}$ has been named as the Benjamin-Feir index $[52,53]$ and it has been shown that when $\epsilon$ is large then the evolution will eventually lead to the formation of rogue waves with a probability larger than the expected from a Rayleigh distribution of the envelope.

For a given value of $\epsilon$ the dynamics is unique, i.e., if we assume that waves propagate according to the NLSE, we expect to observe the same phenomenology in the optical fiber and in the water tank. Varying the power $P_{0}=\left\langle\left|A_{0}\right|^{2}\right\rangle$ and accordingly the spectral width $\Delta \omega=2 \pi \Delta f$ allows one to change the nonlinear length while keeping constant $\epsilon$. This procedure can be used to obtain the desired nonlinear length at the end of the fiber, while keeping unchanged the dynamics. The last helpful ingredient for the comparison of the two experiments is the introduction of the time scale $\tau$ of the coherent structures such as solitons that are solutions of the NLSE. This is obtained by balancing the nonlinear and dispersive terms in Eq. (1):

$$
\tau=\sqrt{\frac{\left|\beta_{2}\right|}{2\left\langle\left|A_{0}\right|^{2}\right\rangle \chi^{(3)}}} .
$$

By considering this quantity as the time unit, we will be able to use dimensionless frequencies in the comparison of the spectra from the optical and hydrodynamical experiments.

\section{STATISTICAL PROPERTY OF RANDOM WAVES: SOME BASICS PROPERTIES}

If one assumes that the Fourier phases of the surface elevation or the electric field are uniformly distributed, then the probability density function (PDF) of the field is Gaussian. In optics the measurement of the electric field in time is not feasible and only the envelope can be measured (see Refs. [30,31] for new developments). To make comparison between optics and hydrodynamics, it is then necessary to build the envelope from the surface elevation. The procedure is well known in the literature (see, for example, Ref. [54]) and consists in constructing a synthetic field, $\tilde{\eta}(t)$ (we consider it at fix $z$ ) that is orthogonal to the $\eta(t)$ and build the auxiliary complex variable $g(t)=\eta(t)+i \tilde{\eta}(t)$. The variable $\tilde{\eta}(t)$ can be computed from the Hilbert transform, which rotate the Fourier coefficients of $\eta(t)$ by $-\pi / 2$ for positive frequencies and $\pi / 2$ for negative frequencies. The modulus of $g(t)$ corresponds to the modulus of $A(t)$. Now assuming that $\eta(t)$ is the superposition of sinusoidal waves with random phases, then its probability density function, $p(\eta)$, is Gaussian:

$$
p(\eta)=\frac{1}{\sigma \sqrt{2 \pi}} \exp \left[-\eta^{2} /\left(2 \sigma^{2}\right)\right]
$$

where $\eta(t)$ and $\tilde{\eta}(t)$ are two random variables.

Assuming that they are independent variables with the same variance,

then the joint probability density function is given by

$$
p(\eta, \tilde{\eta})=p(\eta) p(\tilde{\eta})=\frac{1}{\sigma^{2} 2 \pi} \exp \left[-\left(\eta^{2}+\tilde{\eta}^{2}\right) /\left(2 \sigma^{2}\right)\right] .
$$

Considering the above probability density function in polar coordinates $(|A|, \theta)$ with $|A|=\sqrt{\eta^{2}+\tilde{\eta}^{2}}$ and $\theta$ the phase, and integrating over the values of $\theta$ it is straightforward to show that

$$
p(|A|)=\frac{|A|}{\sigma^{2}} \exp \left[-|A|^{2} /\left(2 \sigma^{2}\right)\right] ;
$$

this is the well-known Rayleigh distribution. The fourth-order normalized moment of such distribution is given by

$$
\kappa=\frac{\left\langle|A|^{4}\right\rangle}{\left\langle|A|^{2}\right\rangle^{2}}=\frac{\int_{0}^{\infty} p(|A|)|A|^{4} d A}{\left(\int_{0}^{\infty} p(|A|)|A|^{2} d A\right)^{2}}=2 .
$$

Here $\kappa$ is one of the observables that will be compared in optical and hydrodynamical experiments. Values larger that 2 imply that the tail of the distribution is fatter than the Rayleigh distribution; i.e., more rogue waves than predicted by linear theory should appear. Starting from Eq. (10), the probability density function for the normalized intensity (or power) $I=$ $|A|^{2} / P_{0}=|A|^{2} /\left(2 \sigma^{2}\right)$ [see Eq. (3)] can be derived:

$$
p(I)=\exp [-I]
$$

The exponential distribution $p(I)$ will be our reference one, when comparing the optical and the hydrodynamical results.

In the typical experiments that will be discussed, at the beginning of the wave tank or optical fiber, we prescribe a spectral shape and random phases. Therefore, very close to the inlet we expect to see $\kappa \simeq 2$ and an exponential distribution for the intensity. Afterwards, waves travel along the tank and fiber and, because of the nonlinearity, their spectrum changes and the statistical distribution of the wave intensity change as well [5], as we be discussed below.

\section{THE JONSWAP SPECTRUM}

The Joint North Sea Wave Project took place in the late 1960s in the North Sea [55]. Wave spectra were computed from 13 measurement stations over $160 \mathrm{~km}$. One of the aims of the experiment was to understand the nonlinear transfer in the energy balance equation. Observations suggested that the spectral shape of the ocean waves depends on the stage of development of the sea state. In Ref. [55] the following parametrization of the frequency wave spectral density was proposed:

$$
S(f)=\frac{\alpha g^{2}}{(2 \pi)^{4} f^{5}} \exp \left[-\frac{5}{4}\left(\frac{f_{0}}{f}\right)^{4}\right] \gamma^{\exp \left[-\frac{\left(f-f_{0}\right)^{2}}{2 \tilde{\sigma}^{2} f_{0}^{2}}\right]},
$$

with $g$ the gravity acceleration, $f_{0}$ the frequency corresponding to the peak of the spectrum, $\tilde{\sigma}=0.07$ if $f \leqslant f_{0}$, and $\tilde{\sigma}=0.09$ if $f>f_{0} ; \alpha$ and $\gamma$ may assume different values depending on the sea state. As $\gamma$ increases, the spectrum becomes more narrow and the power also increases. Large values correspond to young seas, i.e., those for which the phase velocity of the waves is much smaller than the wind speed. The parameter $\alpha$ is related to the power: as it increases, the significant wave height increases as well. As a measure of the width $\Delta f$ of the spectrum, we will use in the following the full width at half maximum.

Here we remark that the dynamics of nonviscous surface gravity waves is fully scalable in the laboratory, in the sense that the dynamics of the ocean waves can be reproduced in a wave tank, provided the adimensional numbers, i.e., the steepness and the relative spectral band width, $\Delta f / f_{0}$, are maintained. Indeed, the JONSWAP spectrum is widely used 


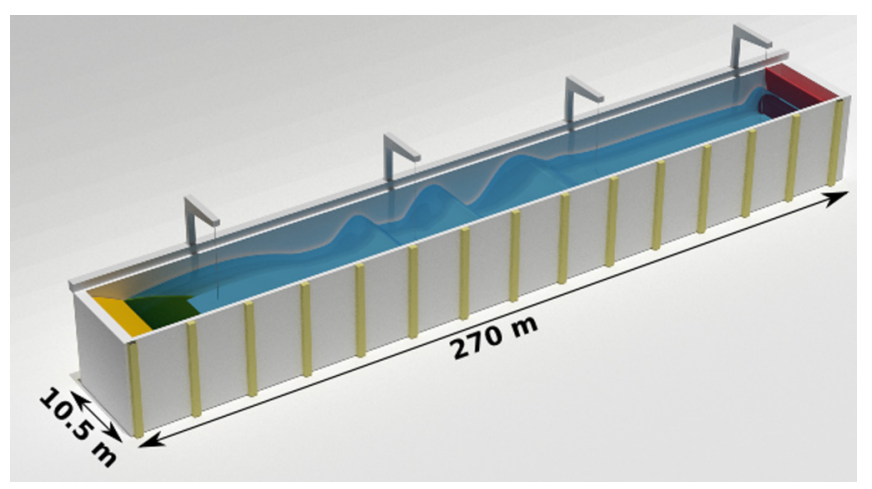

FIG. 1. Sketch of the water wave tank used in the experiment. At one end a fully programmable wave maker is placed to generate waves; at the other end an absorbing beach is placed to minimize reflections. Fifteen probes were placed along the center of the tank at distances of $10,30,35,40,45,60,65,70,75,80,85,115,120,160,200 \mathrm{~m}$ from the wave maker; two extra probes were placed transversally at $z=75$ meters and $z=160 \mathrm{~m}$ to verify if any transverse mode developed in the tank.

for engineering applications. A typical experiment consists in placing a structure (a model of a ship or an offshore platform) at some target location in the tank, launching waves characterized by the JONSWAP spectrum at one end and measuring the response of the structure [56]. Such response is then analyzed for various parameters in the JONSWAP spectrum.

\section{EXPERIMENTAL SETUPS}

\section{A. The hydrodynamical experiment}

The following description is taken from Ref. [45], where the experimental setup is described in detail. The experiment has been performed at Marintek in Trondheim (Norway) in one of the longest existing wave tanks. The length of the flume is $270 \mathrm{~m}$ and its width is $10.5 \mathrm{~m}$ (see Fig. 1). The depth of the tank is $10 \mathrm{~m}$ for the first $85 \mathrm{~m}$, then $5 \mathrm{~m}$ for the rest of the flume. A wave-maker (flap type) located at one end of the tank was used to generate the waves. A sloping beach is located at the far end of the tank opposite the wave maker so that wave reflection is minimized. The wave surface elevation was measured simultaneously by 19 probes placed at different locations along the flume; conductance wave gauges were used.

Once the parameters of the JONSWAP spectrum and the random phases, $\phi_{i}$ are selected, five different time series with a duration of $32 \mathrm{~min}$ each, have been produced as follows:

$$
\eta(t)=\sum_{i=1}^{N} \sqrt{2 S\left(f_{i}\right)} \cos \left(2 \pi f_{i} t+\phi_{i}\right) .
$$

The time series are the input to the software that controls the wave maker. Note that the dynamics is not statistically homogeneous in space but it is statistically stationary in time at each distance from the wave maker.

All experiments were performed with a nominal peak period of the JONSWAP spectrum of $1.5 \mathrm{~s}$. Under these circumstances, the water depth parameter $k_{0} h \simeq 9$, with $k_{0}$ the wave number at the spectral peak and $h$ the water depth, corresponds to the deep water regime. Different values of $\alpha$
TABLE I. Parameters of the JONSWAP spectrum for the two hydrodynamical experiments.

\begin{tabular}{lcccccc}
\hline \hline & $\alpha$ & $\gamma$ & $f_{0}(\mathrm{~Hz})$ & $\Delta f / f_{0}$ & $H_{s}(\mathrm{~cm})$ & $H_{s} k_{0} / 2$ \\
\hline Run $A$ & 0.0112 & 4.8 & 0.667 & 0.178 & 14 & 0.12 \\
Run $B$ & 0.0113 & 8 & 0.667 & 0.154 & 17 & 0.15 \\
\hline \hline
\end{tabular}

and $\gamma$ were selected to have two different values of spectral bandwidth and significant wave height. In Table I we report the parameters of the two experiments, run $A$ and run $B$, here considered.

Here we specify that the parameters contained in the table are the ones obtained by a fitting of the spectrum measured at the first wave gauge, i.e., at $10 \mathrm{~m}$ from the wave maker (the parameters are slightly different from the nominal ones that are specified to the programmable wave maker). Therefore, experiments in optics and numerical computations will all start from a JONSWAP spectrum with random phases with the parameters measured at $10 \mathrm{~m}$ from the wave maker. With such choice we define the new zero coordinate, $z=0$, of our experiment to be located at $10 \mathrm{~m}$ from the wave maker.

As mentioned in the Sec. II, for a correct comparison between the hydrodynamical and the optical experiments it is of paramount importance to introduce the ratio between the linear to nonlinear lengths. Using Eqs. (4), $\beta_{2 \text {,hydro }}=2 / \mathrm{g}$, $\chi_{\text {hydro }}^{(3)}=k_{0}^{3}$, and the dispersion relation of deep water waves $\omega_{0}^{2}=g k_{0}$, one finds for the hydrodynamical experiment that linear and nonlinear lengths are

$$
z_{\operatorname{lin}}=\frac{g}{\Delta \omega^{2}}=\frac{\omega_{0}^{2}}{k_{0} \Delta \omega^{2}}=\frac{f_{0}^{2}}{k_{0} \Delta f^{2}}
$$

and

$$
z_{\text {nlin }}=\frac{1}{k_{0}^{3}\left\langle\left|A_{0}\right|^{2}\right\rangle}=\frac{8}{k_{0}^{3} H_{s}^{2}},
$$

therefore

$$
\epsilon=\frac{z_{\text {lin }}}{z_{\text {nlin }}}=\frac{k_{0}^{2}\left\langle\left|A_{0}\right|^{2}\right\rangle}{\left(\Delta f / f_{0}\right)^{2}},
$$

i.e., the ratio between the square of the steepness and the square of the spectral bandwidth of the initial condition; see Ref. [53]. Comparison between the optical and hydrodynamical experiments will be made by introducing the nondimensional coordinate $z=z^{\prime} / z_{\text {nlin }}$ with $z^{\prime}$ the distance expressed in meters from the first probe. In Table II, the linear and the nonlinear lengths, their ratio, and $z_{\max }=z_{\max }^{\prime} / z_{\text {nlin }}$, with $z_{\max }^{\prime}$ the distance of the last probe from the first one, are reported.

TABLE II. Length scales and nonlinear parameters for the two hydrodynamical experiments.

\begin{tabular}{lcccc}
\hline \hline & $z_{\text {lin }}(\mathrm{m})$ & $z_{\text {nlin }}(\mathrm{m})$ & $\epsilon=z_{\text {lin }} / z_{\text {nlin }}$ & $z_{\text {max }}^{\prime} / z_{\text {nlin }}$ \\
\hline Run $A$ & 17.5 & 71.1 & $\sim 0.25$ & 2.67 \\
Run $B$ & 23.5 & 48.2 & $\sim 0.50$ & 3.94 \\
\hline \hline
\end{tabular}




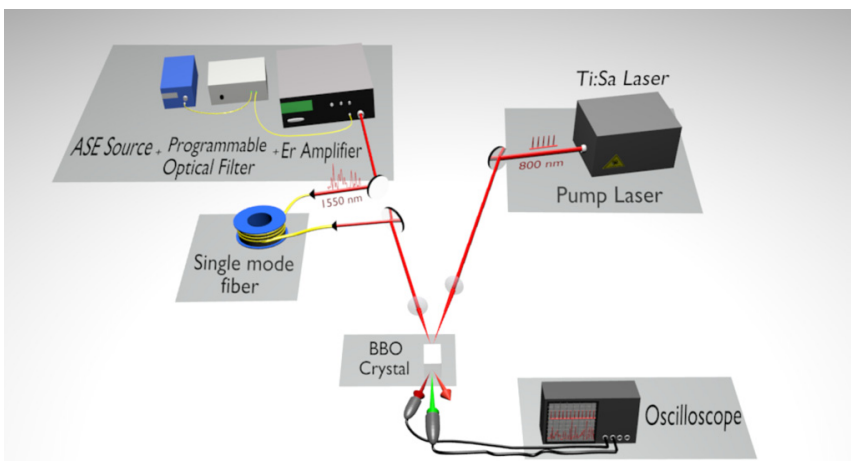

FIG. 2. Optical sampling of the partially coherent wave fluctuating with time (the signal) is achieved from sum frequency generation (SFG). Green pulses are generated at $\lambda=529 \mathrm{~nm}$ from the interaction of the signal with femtosecond pump pulses inside a $\chi^{(2)}$ crystal. The $140 \mathrm{fs}$ pump pulses are emitted by mode-locked laser at $\lambda_{p}=$ $800 \mathrm{~nm}$. The partially coherent wave is emitted by an ASE source at $\lambda_{s}=1562 \mathrm{~nm}$ and is amplified by an Erbium fiber amplifier. Statistics of partially coherent light is measured from the SFG process either directly at the output of the laser or after propagation inside an optical fiber.

Considering that the dominant wave generated in the wave tank has a period of $1.5 \mathrm{~s}$, then our statistics can be obtained at each location by averaging over 6400 linear time scales.

\section{B. The optical experiment}

The optical experimental setup is displayed in Fig. 2. It can be divided in three parts: (i) the source of partially coherent waves, (ii) nonlinear propagation in optical fiber, and (iii) optical sampling detection.

(i) The random optical waves source is comparable to the one described in Ref. [30]: the partially coherent light (i.e., the initial condition) is generated by an Erbium fibre broadband amplified spontaneous emission (ASE) source (Highwave), which is spectrally filtered (with adjustable shape and line width) using a programmable optical filter (Waveshaper 1000S, Finisar). The central wavelength is $\lambda \sim 1562 \mathrm{~nm}$ corresponding to a carrier wave frequency $f_{0 \text {,optics }}=192 \mathrm{THz}$. The output light is then amplified by an Erbium-doped fiber amplifier (Keopsys). Note that numerical simulations have been performed by using initial conditions having both the theoretical JONSWAP spectrum and the spectrum experimentally recorded at the output of the amplifier. These simulations show that the ASE noise of the amplifier does not play any significant role in the dynamics nor in the statistics of the partially coherent wave measured at the output of the fiber.

Using the programmable optical filter, the optical spectrum of the partially coherent light emitted by the amplifier is precisely designed to assume a JONSWAP shape. Note that this step is not straightforward because of the amplification and several empirical feedback loops are necessary to converge. The red line in Fig. 3 is a typical spectrum measured with an optical spectrum analyzer at the output of the random source. Our setup allows us to obtain initial optical spectrum that is very close to the ideal JONSWAP spectrum (black dashed
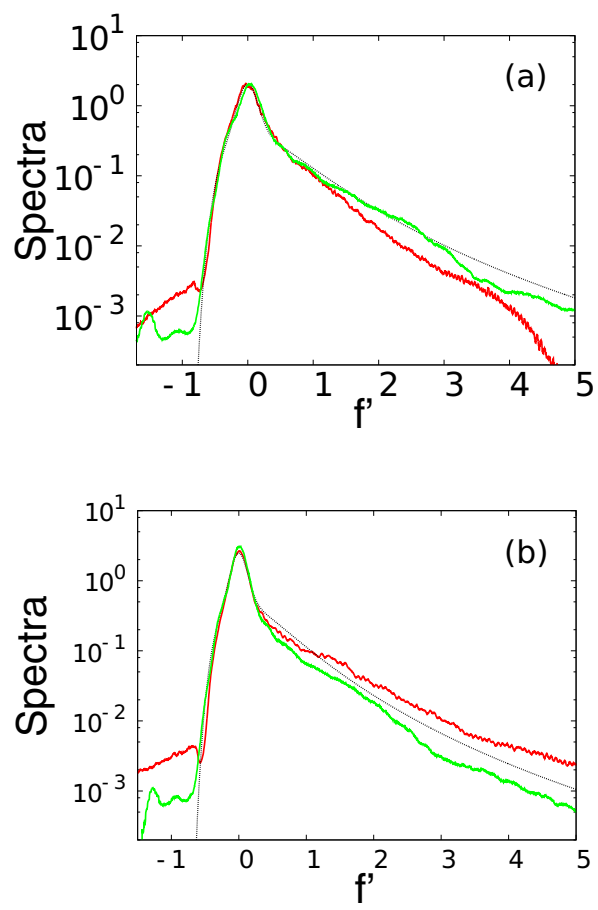

FIG. 3. Spectral power density at $z=0$ in the optical fiber, red (gray) line, and in the water tank, green (light gray) line, for run $A$ (a) and $B$ (b). The JONSWAP spectrum (black line) with parameters taken from Table I is also shown. The frequency $f^{\prime}$ is normalized by using Eq. (20).

lines) and to the spectrum of water waves measured at the first gauge of the water tank (blue line).

(ii) The amplified random light is launched into standard single-mode fibers (SMF28) having different lengths $z_{\text {fiber }}^{\prime}$. The wavelength $\lambda \sim 1562 \mathrm{~nm}$ of random light falls into the anomalous (focusing) regime of dispersion of the fiber that has a group velocity dispersion coefficient $\beta_{2 \text {,iber }}=-22 \mathrm{ps}^{2} \mathrm{~km}^{-1}$ and a Kerr coefficient $\chi_{\text {fiber }}^{(3)} \simeq 1.3 \mathrm{~W}^{-1} \mathrm{~km}^{-1}$

(iii) The statistics of optical power is measured at the input and at the output ends of the optical fiber with the optical sampling setup described in Ref. [29]. Sum frequency generation (SFG) between the random waves under study and femtosecond pump pulses is obtained at a wavelength $\simeq 529 \mathrm{~nm}$ in a BBO crystal. The 140-fs-long pump pulses are emitted by a mode-locked Ti:Sa laser (Coherent Cameleon ultra II) at $800 \mathrm{~nm}$ with a repetition rate of $80 \mathrm{MHz}$. The BBO crystal has $8 \mathrm{~mm}$ length and is cut for noncollinear type I SFG $\left(\theta=24.2^{\circ}, \phi=90^{\circ}\right.$, external angle between pump and signal $\left.=12.5^{\circ}\right)$. By ensuring that the typical power of random waves $(\sim 2 \mathrm{~W})$ is much weaker than the peak power $\left(\sim 4.10^{5} \mathrm{~W}\right)$ of the pump pulses, the energy of each SFG pulse is proportional to the instantaneous optical power $|A|^{2}$ carried by the random waves [57]. The short green pulses are observed by using a highly sensitive photodiode (MenloSystem FPD310-FV) having a gain of $\simeq 10^{4}$ and a rise time of $0.7 \mathrm{~ns}$. We record the output of the photodiode with a fast oscilloscope (Lecroy WaveRunner 104MXi-A, bandwidth $1 \mathrm{GHz}, 10 \mathrm{GS} / \mathrm{s})$. We compute the PDFs of $|A|^{2}$ from typical ensemble of approximately 8 millions measurements of SFG peak powers (see 
Ref. [29] for details). The resolution of the optical sampling technique depends on the duration of the pump pulses and it may also depend on other parameters such as angle between the signal and pump beam (walk off and nonlinear phase matching geometry), group velocity mismatch, etc. In Ref. [29], the temporal resolution has been evaluated to $\sim 250 \mathrm{fs}$, which is smaller than the time scales investigated in this paper.

The key point in the experiments is to use similar values of the relevant parameters both in optical fiber and water tank experiments. This means that for a given initial statistics of water waves and for a given length of the water tank, the reduced parameters $z=z^{\prime} / z_{\text {nlin }}$ and $\epsilon=z_{\text {lin }} / z_{\text {nlin }}$ should be identical in the optical experiment. To compare the statistics of water waves measured at a given length of propagation in the water tank together with the statistics of optical waves at the output of a fiber of length $z_{\text {fiber }}^{\prime}$, we have used the following methodology:

(i) $z=z^{\prime} / z_{\text {nlin }}$ is computed by using Eqs. (4) and (5) and the parameters of the water tank experiments;

(ii) the mean optical power $P_{0}$ is computed by using Eq. (4) and the value of optical nonlinearity $\chi_{\text {fiber }}^{(3)}$;

(iii) the full width at half maximum $\Delta f_{\text {hydro }}$ of the input JONSWAP water waves spectrum is computed from Eq. (13) by using the parameters of the water tank experiments;

(iv) by imposing the same value for $\epsilon$ [Eq. (6)] in optical and hydrodynamical experiments, one obtains the value of the full width at half maximum $\Delta f_{\text {optics }}$ of the input JONSWAP spectrum to be used in the optical experiment:

$$
\Delta f_{\text {optics }}=\mu \Delta f_{\text {hydro }} \text { with } \mu=4 \sqrt{\frac{\chi_{\text {fiber }}^{(3)} P_{0}}{\beta_{2, \text { fiber }} g k_{0}^{3} H_{s}^{2}}} .
$$

Note that $\mu$ represents the ratio between the typical time scales of the structures emerging in water waves and optical fibers experiments and it is of the order $10^{12}$ in our experiments.

(v) Finally, the spectrum $S_{\text {optics }}(f)$ of the partially coherent optical waves is designed by using the programmable optical filter. The optical spectrum follows the JONSWAP spectrum given by the Eq. (13) with the broadening factor $\mu$ :

$$
S_{\text {optics }}\left(f_{\text {optics }}\right)=S\left(\frac{f_{\text {optics }}-f_{0, \text { optics }}}{\mu}+f_{0}\right),
$$

where $f_{0}$ is the central frequency of water waves.

By using the procedure outlined in (i-v), for a given propagation length in water tank and a given value of the significant water wave height, an equivalent optical fiber experiment is performed. Contrary to the so-called cutback technique [47-49], the technique used here (and similar for example to the one used in Ref. [35]) allows us to explore various normalized propagation lengths with only one optical fiber having a fixed length. The key point is to adjust the spectral width and the optical power to change proportionally the number of linear lengths and of nonlinear lengths in the physical length of the optical fiber that is used.

The set of parameters used in the optical fiber experiments and their counterparts in the water tank are displayed in Tables III and IV for run $A$ and run $B$ experiments, respectively. In principle, our scaling technique allows the use of one fiber length to investigate all the normalized lengths of propagation. However, to use easily available optical powers and to keep the
TABLE III. Optical parameters corresponding to the water wave experiment run $A$; see Tables I and II.

\begin{tabular}{lccccc}
\hline \hline$z_{\text {fiber }}^{\prime}(\mathrm{m})$ & $P_{0}(\mathrm{~W})$ & $\Delta f_{\text {opt }}(\mathrm{THz})$ & $z^{\prime} / z_{\text {lin }}$ & $z^{\prime} / z_{\text {nlin }}$ & $z_{\text {hydro }}^{\prime}(\mathrm{m})$ \\
\hline 50 & 2.0 & 0.150 & 0.49 & 0.13 & 10 \\
125 & 1.6 & 0.141 & 1.08 & 0.26 & 20 \\
250 & 1.2 & 0.128 & 1.78 & 0.40 & 30 \\
250 & 1.6 & 0.140 & 2.13 & 0.53 & 40 \\
500 & 1.2 & 0.129 & 3.61 & 0.79 & 60 \\
500 & 1.6 & 0.140 & 4.25 & 1.06 & 80 \\
1000 & 1.2 & 0.130 & 7.33 & 1.59 & 120 \\
1250 & 1.2 & 0.117 & 7.42 & 1.98 & 150 \\
1000 & 2.0 & 0.139 & 8.38 & 2.64 & 200 \\
\hline \hline
\end{tabular}

signal to noise ratio roughly constant (i.e., by avoiding very low power), we have used several lengths of optical fibers. The tables contain the lengths of the different fibers used in the experiments and the corresponding propagation distances in meters achieved in the water wave experiment (last column $z_{\text {hydro }}^{\prime}$ ).

\section{RESULTS AND DISCUSSION}

\section{A. Input spectra}

An important step toward a comparison between the results is to start the hydrodynamical and optical experiment with compatible spectra. To check such compatibility, we find it useful to introduce the following nondimensional frequency:

$$
f^{\prime}=\tau\left(f-f_{0}\right),
$$

where $\tau$ is defined in Eq. (7). In optics $\tau_{\text {optics }}=$ $\sqrt{\left|\beta_{2}\right| /\left(2 \chi^{(3)} P_{0}\right)}$, while in hydrodynamics $\tau_{\text {hydro }}=$ $\sqrt{8 /\left(g k_{0}^{3} H_{s}^{2}\right)}$. In Figs. 3(a) and 3(b) we show the frequency spectra as a function of $f^{\prime}$ for run $A$ and run $B$ at $z^{\prime}=0$; the JONSWAP spectrum with parameters reported in Table $\mathrm{I}$ is also shown. A very good agreement in the initial condition is shown for the most energetic part of the spectrum.

TABLE IV. Optical parameters corresponding to the water wave experiment run $B$; see Tables I and II.

\begin{tabular}{lccccc}
\hline \hline$z_{\text {fiber }}^{\prime}(\mathrm{m})$ & $P_{0}(\mathrm{~W})$ & $\Delta f_{\text {opt }}(\mathrm{THz})$ & $z^{\prime} / z_{\text {lin }}$ & $z^{\prime} / z_{\text {llin }}$ & $z_{\text {hydro }}^{\prime}(\mathrm{m})$ \\
\hline 125 & 1.2 & 0.089 & 0.43 & 0.20 & 10 \\
125 & 2.4 & 0.122 & 0.81 & 0.39 & 20 \\
250 & 1.8 & 0.112 & 1.37 & 0.59 & 30 \\
250 & 2.4 & 0.123 & 1.64 & 0.78 & 40 \\
500 & 1.8 & 0.114 & 2.82 & 1.17 & 60 \\
500 & 2.1 & 0.108 & 2.53 & 1.37 & 70 \\
500 & 2.4 & 0.127 & 3.50 & 1.56 & 80 \\
1000 & 1.3 & 0.084 & 3.06 & 1.66 & 85 \\
1000 & 1.8 & 0.114 & 5.62 & 2.34 & 120 \\
1250 & 1.8 & 0.105 & 5.98 & 2.93 & 150 \\
1000 & 3.0 & 0.138 & 8.26 & 3.90 & 200 \\
\hline \hline
\end{tabular}



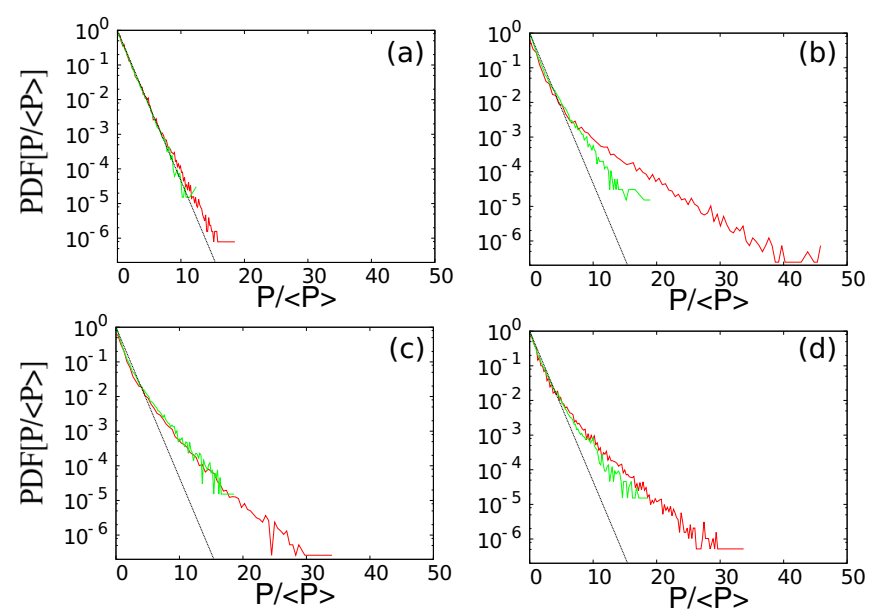

FIG. 4. Probability density function of the normalized intensity, $I$, for the experiments in run $B(\epsilon \sim 0.5$ at different nondimensional distances: (a) $z=z^{\prime} / z_{\text {nlin }}=0$, (b) $z \simeq 0.6$, (c) $z \simeq 1.4$, (d) $z \simeq 4$. The green (light gray) line corresponds to the water wave experiment and the red one (gray) to the optical one. The black line corresponds to the exponential distribution.

\section{B. Statistical properties of the intensity}

\section{The probability density function of the Intensity}

As mentioned in Sec. III we concentrate our analysis on the statistical properties of the normalized intensity of the wave field: $I=|A|^{2} / P_{0}$ in optics and $I=|A|^{2} /\left(2 \sigma^{2}\right)$ in hydrodynamics. We recall that the surface elevation is measured in the hydrodynamical experiment. Therefore, to compute the intensity, the envelope $A$ has to be calculated using the Hilbert transform.

The probability density functions (PDF) of $I$ measured both in the water wave tank and in the optical fiber experiments for a strength of the nonlinearity corresponding to run $B$ are displayed in Figs. 4 for different propagation lengths. As expected, the PDFs of the intensity measured at the beginning of the fiber and tank $\left(z_{\text {fiber }}=z_{\text {hydro }}=0\right)$ are very close to the exponential distribution. This is expected because the waves are generated by using a prescribed spectral shape with random phases. When the length of propagation is sufficiently large, the nonlinearity begins to become important. The statistics of both optical and hydrodynamical intensities deviate from the exponential distribution, displaying heavy tails. Strikingly, for high values of the normalized length (typically $z>2$ ), the PDFs measured in optical fibers and in the water wave tank experiments are remarkably close [see Figs. 4(c) and 4(d)]. For intermediate values of $z \simeq 0.5-1$, the optical waves exhibit stronger deviation from the exponential distribution than the water waves [see Fig. 4(b)]. This issue will be further discussed in Sec. VIC where numerical simulations are reported. It is interesting to note the different probability levels that are achieved in optical fibers and the water tanks. For the hydrodynamics experiments, PDFs are obtained by averaging over five realizations with different phases each lasting 32 min (in between the different realizations, about $30 \mathrm{~min}$ were needed to let the waves damp in the tank). The time needed in the optical experiment to collect the data for a PDF is less
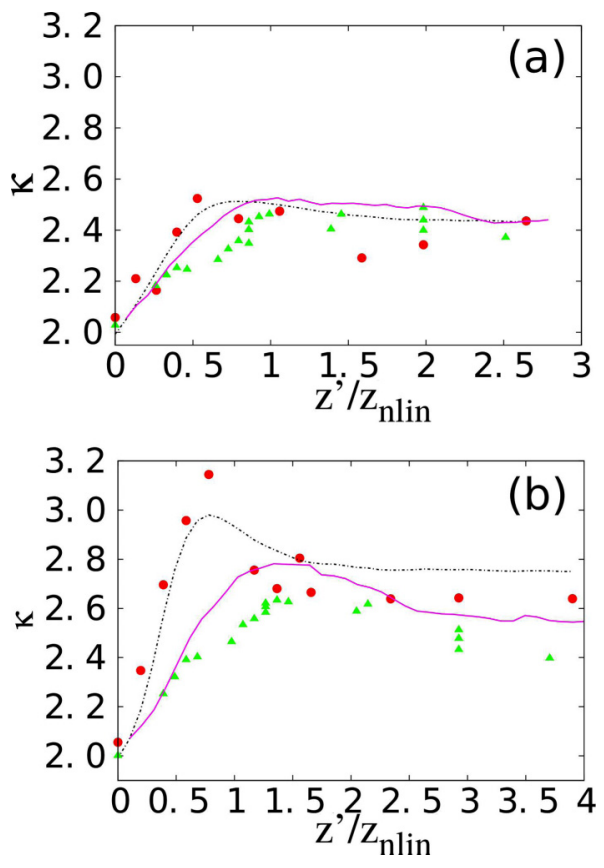

FIG. 5. Fourth-order normalized moment of the wave envelope moment, $\kappa=\left\langle|A|^{4}\right\rangle /\left\langle|A|^{2}\right\rangle^{2}$, as a function of the nondimensional propagation coordinate $z$ : experiments and numerical simulations for run $A$ (panel a) and run $B$ (panel b). Optical experiments (red points), hydrodynamical experiments (green triangles), simulations of the NLSE (dashed line), simulations of Euler equation (magenta line).

than $10 \mathrm{~ms}$ and the probability level achieved is more than one order of magnitude lower than in the water wave experiment.

The PDFs of run $A$ (not reported here) display the same features of those of run $B$, with the only difference being that the level of nonlinearity is lower and the deviations from the exponential distribution are less prominent.

\section{The normalized fourth-order moment of the probability density function of the envelope}

To compare the statistical properties of water and optical waves, we have computed the normalized fourth-order moment $\kappa$, see Eq. (11), of the wave envelope for each experimental parameters as a function of the normalized propagation distance $z=z^{\prime} / z_{\text {nlin }}$. We remark that values larger than 2 imply a departure from linear predictions. In Fig. 5, the fourth-order moment of $|A|$ is plotted as a function of $z$ for hydrodynamics (blue dots) and optical (red dots) tests: the case corresponding to run $A$ is displayed in Fig. 5(a) and the higher nonlinear case, run $B$, is displayed in Fig. 5(b). Numerical simulations of the NLS equation and the Euler equation for water waves are also displayed (see Sec. VIC). For both runs, $A$ and $B, \kappa$ starts from the value of 2 and then grows on a spatial scale of the order of a nonlinear length. Run $B$ is more nonlinear than run $A$ and displays larger deviations from the linear predictions. The optical experiment displays a faster growth of $\kappa$ and it is more consistent with the NLE equation. Both experiments show a maximum value of $\kappa$ at some length after which $\kappa$ is almost constant. A significant overshoot in the evolution of $\kappa$ 
for optical waves around $z=0.7$ in run $B$ [Fig. 5(b)] can be observed, while it is less pronounced for water waves.

In the weaker nonlinear case, run $A$, displayed in Fig. 5(a), the agreement between optical and water waves experiments is strikingly good. The evolution of $\kappa$ is a little bit slower for water waves but both cases seem to reach a stationary state $\kappa \simeq 2.4$ around $z=1$. Note that the point $z=1.5$ has been recorded with a SMF fiber of a different spool and we interpret the decreasing of $\kappa$ for this point as the influence of a slight change in the Kerr effect coefficient.

Finally, we want to emphasize that despite some differences between the optical and hydrodynamical results, the agreement between the two completely different experiments is remarkable.

\section{Numerical simulations}

To interpret the differences between optical and waters waves statistics, we have performed numerical simulations using two different models: the NLSE and the Euler equations for water waves has been solved numerically.

\section{NLSE simulations}

In standard optical fibers, the power losses are very low ( $\simeq 0.18 \mathrm{~dB} / \mathrm{km}$, i.e., $2 \%$ in $500 \mathrm{~m}$, for example). Experiments performed with partially coherent waves having a narrow spectrum and a central wavelength far from the zero dispersion wavelength are known to be well described by the NLSE $[30,58]$. Note that mean powers used in optical experiments are relatively low and that we have checked from optical spectra measurement that stimulated Raman scattering can be neglected. Our experiments involving time scales of a few picoseconds are typically well described by the NLSE as long as $z / z_{\text {nlin }}<15$; see Ref. [58].

Equation (1) has been solved numerically using periodic boundary conditions in time. We have considered a complex field $A(z=0, t)$ having a JONSWAP optical power spectrum, Eq. (19), and random phases. Statistical properties of the waves have been computed from Monte Carlo simulations made with an ensemble of $10^{4}$ realizations characterized by different random phases for the initial condition. The normalized fourthorder moment $\kappa$ is plotted with black lines in the Fig. 5 together with the optical fiber and water tank experiments. The agreement between experiments performed in optical fibers and numerical simulations is overall very good.

Concerning the comparison of the simulations with the hydrodynamical experiment, it is clear especially from Fig. 5(b) that the dynamics of water waves is slower than the numerical predictions. This result is well known and already documented, for example, in Ref. [59], and it is basically due to the fact that the NLS theory does not properly describe short groups, strong nonlinearity, and white capping (sporadically observed in the experiment).

In conclusion, the evolution of $\kappa$ as a function of $z=z^{\prime} / z_{\text {nlin }}$ computed from NLSE reproduces better the optical rather than the hydrodynamical experiment. This is not a surprise because the optical fiber has been designed to be properly described by the NLSE, while there is no possibility to modify the medium to change the dispersion and the nonlinearity to match better the equation in the water wave context.

\section{Euler simulations}

In Fig. 5 we have also reported numerical simulations of the Euler equations for water waves. The fluid has been considered as inviscid, incompressible, and irrotational. The set of equations for the surface elevation $\eta(x, t)$ and for the velocity potential, $\psi(x, t)$, on the free surface that have been solved are the following:

$$
\begin{aligned}
& \frac{\partial \eta}{\partial t}+\frac{\partial \psi}{\partial x} \frac{\partial \eta}{\partial x}-w\left[1+\left(\frac{\partial \eta}{\partial x}\right)^{2}\right]=0 \\
& \frac{\partial \psi}{\partial t}+g \eta+\frac{1}{2}\left(\frac{\partial \psi}{\partial x}\right)^{2}-\frac{1}{2} w^{2}\left[1+\left(\frac{\partial \eta}{\partial x}\right)^{2}\right]=0
\end{aligned}
$$

where $w$ is the vertical velocity computed on the free surface. Its calculation in principle requires the knowledge of the velocity field in the whole domain (under the free surface). To address this problem we have used the so called higher order spectral method (see Refs. [60,61]) by which an iterative procedure is used for expressing the vertical velocity as a function of the surface elevation and the velocity potential on the surface. When the iterative scheme is truncated adequately the system is Hamiltonian. The system is solved as evolution equations in time with periodic boundary condition in space. To compare the results with the experiments, the group velocity is then used to convert time to space. The numerical method does not allow for wave breaking. The initial conditions are provided by the JONSWAP spectrum (once converted in a wave numbers spectrum) with random phases for the surface elevation and the velocity potential is then calculated assuming linear theory (see Ref. [62] for details).

The results obtained from the simulations are displayed in Fig. 5. The evolution of $\kappa$ provided by the Euler equations shows a much better agreement to the hydrodynamical experiment with respect to the NLSE simulations. The values of $\kappa$ are just slightly overestimated with respect to the experimental results, especially for run $B$. We believe that such effect is largely due to the impossibility of the model to reproduce the wave breaking phenomenon visually observed in the tank. Any damping such as the one due to the presence of lateral walls is also not included in the simulations. The asymptotic state reached in the simulations is slightly larger than the one obtained in the experiments.

\section{CONCLUSIONS}

Starting from the pioneering work of Solli et al. [33], the concept of rogue waves has been introduced in the optical community. Since then, a lot of work has been done, trying to deepen the analogies between optical and hydrodynamical rogue waves [63]. The bridge for the analogy finds its roots in the universality of the NLS equation [40] and its capability of describing weakly nonlinear dispersive waves in different contexts. Indeed, exact breather solutions of the NLSE have been reproduced with some degree of success both in hydrodynamics and optics [36]. However, apart from very special conditions, ocean waves cannot be considered as a small perturbation of a coherent wave. Measurements during field experiments of the surface elevation show that ocean waves 
are characterized by a finite-width spectrum whose phases are hardly distinguishable from a set of random phases.

In the present paper an optical experiment has been devised to properly reproduce the statistical properties of gravity waves measured in a long wave tank with initial conditions characterized by a JONSWAP spectrum and random phases. The key role for designing properly the optical experiment is played by the NLSE. Indeed, out of the equation nondimensional distances and nonlinear parameters can be derived and used for comparing the experiments. Two sets of experiments have been performed characterized by two different ratios between linear and nonlinear propagation distances. In the context of ocean waves, such a ratio is nothing but the square of the so-called Benjamin-Feir index defined in Refs. [2,53]. Larger values of such index in the initial condition leads to the formation of more rogue waves than the linear theory would predict and consequently the formation of heavy tails in the probability distribution of the wave intensity. Such behavior has been observed in the optical experiment.

A one-to-one comparison between the hydrodynamical and the optical experiments has been performed. The focus has been on the probability density function of the wave intensity and on the evolution of the fourth-order moment of the probability density function of the wave envelope. While in a wave tank measurements of the surface elevation along the tank are always possible, they can be done only at its end in an optical fiber. An appropriate technique based once more on the NLSE equation has allowed us to perform measurements at different propagation distances using the same fiber by changing the width of the initial spectrum and its power.

Having in mind that the optical and hydrodynamical experiments are completely different, a quantitative comparison between them shows a remarkable agreement. Heavy tails and deviation from linear statistics are observed in both experiments at common nondimensional propagation distances. Faster evolution of the dynamics is observed in the optical experiment. As mentioned, this is due to the fact that the latter has been designed to match properly the NLSE equation, which is not an optimal model for water waves. Numerical simulations of the NLSE confirm our findings. A numerical study of the Euler equation has been performed and the results show a better agreement than the NLSE with the hydrodynamical experiment. Here we stress that propagation in both experiments is one dimensional. Recently, it has been found that two-dimensionality may play an important role in the statistical properties of ocean waves [64-66]. We hope that our success in the comparison will trigger new work in the even more complicated optical set-ups ruled by two-dimensional propagation.

\section{ACKNOWLEDGMENTS}

This work has been partially supported by the French Agence Nationale de la Recherche through the LABEX CEMPI project (Grant No. ANR-11-LABX-0007) and the OPTIROC project (Grant No. ANR-12-BS04-0011 OPTIROC) as well as by the Ministry of Higher Education and Research, Nord-Pas de Calais Regional Council and European Regional Development Fund (ERDF) through the Contrat de Projets Etat-Region (CPER Photonics for Society P4S). M.O. has been funded by Progetto di Ricerca d'Ateneo CSTO160004. The authors are grateful to E. Courtade, F. Anquez, J. Pesez, and the Biophysics of Cellular Stress Response group of the PhLAM for providing the femtosecond laser used in optical sampling. Dr. B. Giulinico is acknowledged for discussion.
[1] G. Komen, L. Cavaleri, M. Donelan, K. Hasselmann, H. Hasselmann, and P. Janssen, Dynamics and Modeling of Ocean Waves (Cambridge University Press, Cambridge, 1994).

[2] P. Janssen, The Interaction of Ocean Waves and Wind (Cambridge University Press, Cambridge, 2004), p. 379.

[3] C. Kharif, E. Pelinovsky, and A. Slunyaev, Rogue Waves in the Ocean (Springer Verlag, Berlin, 2009).

[4] M. Onorato, S. Residori, U. Bortolozzo, A. Montina, and F. Arecchi, Phys. Rep. 528, 47 (2013).

[5] M. Onorato, D. Proment, G. El, S. Randoux, and P. Suret, Phys. Lett. A 380, 3173 (2016).

[6] F. Fedele, J. Brennan, S. P. De León, J. Dudley, and F. Dias, Sci. Rep. 6, 27715 (2016).

[7] N. Akhmediev, A. Ankiewicz, and M. Taki, Phys. Lett. A 373, 675 (2009).

[8] J. M. Dudley, F. Dias, M. Erkintalo, and G. Genty, Nat. Photon. 8, 755 (2014).

[9] M. Onorato, S. Rsidori, and F. Baronio (eds.), Rogue and Shock Waves in Nonlinear Dispersive Media, Lecture Notes in Physics Vol. 926 (Springer, Heidelberg, 2016).

[10] F. T. Arecchi, U. Bortolozzo, A. Montina, and S. Residori, Phys. Rev. Lett. 106, 153901 (2011).

[11] M. Leonetti and C. Conti, Appl. Phys. Lett. 106, 254103 (2015).
[12] K. Trulsen and K. B. Dysthe, in Proceedings of the 21st Symposium on Naval Hydrodynamics (National Academy Press, Washington, DC, 1997), pp. 550-560.

[13] K. L. Henderson, D. H. Peregrine, and J. W. Dold, Wave Motion 29, 341 (1999).

[14] N. Akhmediev, V. Eleonskii, and N. Kulagin, Theor. Math. Phys. 72, 809 (1987).

[15] E. Kuznetsov, Akademiia Nauk SSSR Doklady 236, 575 (1977).

[16] D. Peregrine, J. Aust. Math. Soc. Series B 25, 16 (1983).

[17] N. Akhmediev, J. M. Dudley, D. R. Solli, and S. K. Turitsyn, J. Opt. 15, 060201 (2013).

[18] D. R. Solli, C. Ropers, and B. Jalali, Phys. Rev. Lett. 101, 233902 (2008).

[19] M. Erkintalo, G. Genty, and J. M. Dudley, Opt. Lett. 34, 2468 (2009).

[20] B. Kibler, C. Finot, and J. M. Dudley, Eur. Phys. J.: Spec. Top. 173, 289 (2009).

[21] A. Mussot, A. Kudlinski, M. Kolobov, E. Louvergneaux, M. Douay, and M. Taki, Opt. Express 17, 17010 (2009).

[22] A. Montina, U. Bortolozzo, S. Residori, and F. T. Arecchi, Phys. Rev. Lett. 103, 173901 (2009).

[23] D. Pierangeli, F. Di Mei, C. Conti, A. J. Agranat, and E. DelRe, Phys. Rev. Lett. 115, 093901 (2015). 
[24] C. Bonatto, M. Feyereisen, S. Barland, M. Giudici, C. Masoller, J. R. Rios Leite, and J. R. Tredicce, Phys. Rev. Lett. 107, 053901 (2011).

[25] C. Lecaplain, P. Grelu, J. M. Soto-Crespo, and N. Akhmediev, Phys. Rev. Lett. 108, 233901 (2012).

[26] J. Kasparian, P. Béjot, J.-P. Wolf, and J. M. Dudley, Opt. Express 17, 12070 (2009).

[27] K. Hammani, C. Finot, J. M. Dudley, and G. Millot, Opt. Express 16, 16467 (2008).

[28] S. Randoux and P. Suret, Opt. Lett. 37, 500 (2012).

[29] P. Walczak, S. Randoux, and P. Suret, Phys. Rev. Lett. 114, 143903 (2015).

[30] P. Suret, R. E. Koussaifi, A. Tikan, C. Evain, S. Randoux, C. Szwaj, and S. Bielawski, Nat. Commun. 7, 13136 (2016).

[31] M. Närhi, B. Wetzel, C. Billet, S. Toenger, T. Sylvestre, J.-M. Merolla, R. Morandotti, F. Dias, G. Genty, and J. M. Dudley, Nat. Commun. 7, 13675 (2016).

[32] C. Rimoldi, S. Barland, F. Prati, and G. Tissoni, Phys. Rev. A 95, 023841 (2017).

[33] D. Solli, C. Ropers, P. Koonath, and B. Jalali, Nature 450, 1054 (2007).

[34] B. Kibler, J. Fatome, C. Finot, G. Millot, F. Dias, G. Genty, N. Akhmediev, and J. Dudley, Nat. Phys. 6, 790 (2010).

[35] B. Kibler, J. Fatome, C. Finot, G. Millot, G. Genty, B. Wetzel, N. Akhmediev, F. Dias, and J. Dudley, Sci. Rep. 2, 463 (2012).

[36] B. Kibler, A. Chabchoub, A. Gelash, N. Akhmediev, and V. E. Zakharov, Phys. Rev. X 5, 041026 (2015).

[37] B. Kibler, J. Fatome, C. Finot, and G. Millot, in Rogue and Shock Waves in Nonlinear Dispersive Media (Springer, Berlin, 2016), pp. 89-115.

[38] A. Chabchoub, N. P. Hoffmann, and N. Akhmediev, Phys. Rev. Lett. 106, 204502 (2011).

[39] L. Shemer and L. Alperovich, Phys. Fluids 25, 051701 (2013).

[40] A. Chabchoub, B. Kibler, C. Finot, G. Millot, M. Onorato, J. Dudley, and A. Babanin, Ann. Phys. 361, 490 (2015).

[41] M. Onorato, F. Baronio, M. Conforti, A. Chabchoub, P. Suret, and S. Randoux, in Rogue and Shock Waves in Nonlinear Dispersive Media (Springer, Berlin, 2016), pp. 1-22.

[42] B. Jalali, D. Solli, K. Goda, K. Tsia, and C. Ropers, Eur. Phys. J.: Spec. Top. 185, 145 (2010).

[43] D. Solli, G. Herink, B. Jalali, and C. Ropers, Nat. Photon. 6, 463 (2012).

[44] L. Mandel and E. Wolf, Optical Coherence and Quantum Optics (Cambridge University Press, Cambridge, UK, 1995), p. 1192.

[45] M. Onorato, A. Osborne, M. Serio, L. Cavaleri, C. Brandini, and C. T. Stansberg, Eur. J. Mech. B/Fluids 25, 586 (2006).
[46] M. Onorato, A. R. Osborne, M. Serio, L. Cavaleri, C. Brandini, and C. T. Stansberg, Phys. Rev. E 70, 067302 (2004).

[47] B. Barviau, S. Randoux, and P. Suret, Opt. Lett. 31, 1696 (2006).

[48] A. Mussot, A. Kudlinski, M. Droques, P. Szriftgiser, and N. Akhmediev, Phys. Rev. X 4, 011054 (2014).

[49] A. Tikan, C. Billet, G. El, A. Tovbis, M. Bertola, T. Sylvestre, F. Gustave, S. Randoux, G. Genty, P. Suret, and J. M. Dudley, Phys. Rev. Lett. 119, 033901 (2017).

[50] S. Randoux, P. Walczak, M. Onorato, and P. Suret, Phys. Rev. Lett. 113, 113902 (2014).

[51] S. Randoux, P. Walczak, M. Onorato, and P. Suret, Physica D: Nonlin. Phenom. 333, 323 (2016).

[52] P. A. E. M. Janssen, J. Phys. Oceanogr. 33, 863 (2003).

[53] M. Onorato, A. R. Osborne, M. Serio, and S. Bertone, Phys. Rev. Lett. 86, 5831 (2001).

[54] P. A. Janssen, J. Fluid Mech. 759, 236 (2014).

[55] K. Hasselmann, T. Barnett, E. Bouws, H. Carlson, D. Cartwright, K. Enke, J. Ewing, H. Gienapp, D. Hasselmann, P. Kruseman et al., Measurements of wind-wave growth and swell decay during the Joint North Sea Wave Project (JONSWAP), Tech. Rep. (Deutches Hydrographisches Institut, 1973).

[56] Y. Goda, Random Seas and Design on Marine Structures, Advanced Series on Ocean Engineering Vol. 15 (World Scientific, Singapore, 2000), p. 443.

[57] R. W. Boyd, Nonlinear Optics (Academic Press, San Diego, 1992).

[58] G. P. Agrawal, Nonlinear Fiber Optics, 3rd ed., Optics and Photonics (Academic Press, San Diego, 2001).

[59] H. Zhang, C. Guedes Soares, and M. Onorato, Ocean Eng. 89, 1 (2014).

[60] D. G. Dommermuth and D. K. Yue, J. Fluid Mech. 184, 267 (1987).

[61] B. J. West, K. A. Brueckner, R. S. Janda, D. M. Milder, and R. L. Milton, J. Geophys. Res. 92, 11803 (1987).

[62] A. Toffoli, O. Gramstad, K. Trulsen, J. Monbaliu, E. M. BitnerGregersen, and M. Onorato, J. Fluid Mech. 664, 313 (2010).

[63] N. Akhmediev, B. Kibler, F. Baronio, M. Belić, W.-P. Zhong, Y. Zhang, W. Chang, J. M. Soto-Crespo, P. Vouzas, P. Grelu et al., J. Opt. 18, 063001 (2016).

[64] M. Onorato, T. Waseda, A. Toffoli, L. Cavaleri, O. Gramstad, P. A. E. M. Janssen, T. Kinoshita, J. Monbaliu, N. Mori, A. R. Osborne, M. Serio, C. T. Stansberg, H. Tamura, and K. Trulsen, Phys. Rev. Lett. 102, 114502 (2009).

[65] T. Waseda, T. Kinoshita, and H. Tamura, J. Phys. Oceanogr. 39, 621 (2009).

[66] N. Mori, M. Onorato, and P. A. E. M. Janssen, J. Phys. Oceanogr. 41, 1484 (2011). 